\title{
MULTI-DOMAIN BOUNDARY ELEMENT METHOD FOR AXISYMMETRIC PROBLEMS IN POTENTIAL THEORY AND LINEAR ISOTROPIC ELASTICITY
}

\author{
VASYL V. GNITKO ${ }^{1}$, KYRYL G. DEGTYARIOV ${ }^{1}$, ARTEM A. KARAIEV ${ }^{2}$ \& ELENA A. STRELNIKOVA ${ }^{1,2^{*}}$ \\ ${ }^{1}$ A.N. Podgorny Institute for Mechanical Engineering Problems of the Ukrainian Academy of Sciences, Ukraine \\ ${ }^{2}$ V.N. Karazin Kharkiv National University, Ukraine
}

\begin{abstract}
The paper presents an approach based on reduced boundary element methods to resolve axisymmetric problems in potential and linear isotropic elasticity theories. The singular integral equations for these problems are received using fundamental solutions. Initially three-dimensional problems expressed in Cartesian coordinates are transformed to cylindrical ones and integrated with respect to the circumference coordinate. So the three-dimensional axisymmetric problems are reduced to systems of singular integral equations requiring the evaluation of linear integrals only. The fundamental solutions and their derivatives are expressed in terms of complete elliptic integrals. The effective algorithm for treatment of the singular integrals is proposed. The multi-domain boundary element method is applied for the numerical simulation. As examples, the following problems are considered: fluid induced vibrations of a compound cylindrical-spherical elastic shell partially filled with an ideal incompressible liquid, and axisymmetric elasticity problems for an isotropic body with rigid or elastic circular cylindrical inclusions.

Keywords: axisymmetric problems, potential theory, linear isotropic elasticity, multi-domain boundary element method, compound cylindrical-spherical shells.
\end{abstract}

\section{INTRODUCTION}

The boundary element method (BEM) has been used in potential theory and elasticity problems for decades. In order to solve the large-scale problems, researchers developed the multi-domain boundary element method (MDBEM). The multi-domain collocation strategy was briefly introduced by Brebbia et al. in [1], and then developed by Wang and Gao [2], Kane et al. [3], Zhang et al. [4] and Gao and Yang [5]. In MDBEM, the computational domain is divided into a number of sub-domains and the BEM algebraic equations are formulated for each sub-domain. Then the global system of equations is obtained by assembling results of all sub-domains using the equilibrium conditions in common interface nodes. The global system matrix based on MDBEM has a blocked and sparse character, and therefore the well-developed solvers for sparse systems can be employed to solve it. The use of MDBEM not only improve both the efficiency and computational speed, but also can solve fracture problems by dividing sub-domains along crack surfaces [4], multi-media problems [5], and problems for tanks with baffles [6] by dividing sub-domains along interfaces and baffle surfaces. In many engineering problems both geometry and boundary conditions are axisymmetric about some axis. BEM was initially developed for potential theory problems [1] and later extended to elasticity problems [7] due to analogy between fundamental solutions of both theories. The BEM for axisymmetric elasticity was first applied by Cruse et al. [8], using the fundamental solution obtained by Kermanidis [9]. Further BEM applications to axisymmetric problems in elasticity, thermo-elasticity, and fracture mechanics have been developed by Bakr [10]. But application of multi-domain BEM to axisymmetric problems is still not fully described in the literature.

*ORCID: http://orcid.org/0000-0003-0707-7214 


\section{PROBLEM STATEMENTS}

In this paper, we describe applications of the MDBEM to solutions of the problems in potential theory and linear isotropic elasticity. The emphasis is on problems in which the region and the boundary conditions are axisymmetric. In this case, the solution is independent of the angular coordinate $\theta$, and the three-dimensional problem is reduced to a two-dimensional problem in the radial coordinate $r$ and the axial coordinate $z$.

Although boundary integral formulations for different partial differential equations will change due to the presence of different Green's functions, the integral equations of potential theory and theory of elasticity are similar. Moreover, the Green's function behaviour at the singular point $P=Q$ is essentially the same for these problems. This allows us to begin with the simplest boundary integral equation, namely the Laplace equation.

Integral equations for linear potential flow problems are formulated through the application of Green's third identity, that represents a harmonic function as the sum of single- and double-layer potentials. Taking the field point to the boundary, an integral equation relating to only boundary values and normal derivatives of the harmonic function is obtained. Its counterpart in elasticity is Somigliana's identity.

\subsection{Liquid induced vibrations of a compound cylindrical-spherical elastic shell}

Containment shell structures have been the subject of intense research over the last 50 years, and the literature in this area is substantial. Shells composed of cylindrical and spherical parts in interaction with a fluid have received a little attention in scientific literature in spite of the importance of such thin walled structures in a number of different engineering branches. They are often used as tanks for liquid and gas storage, and as pressure vessels. They also appear as components of aerospace and marine vehicles, such as rocket and balloon skins, and submarine hulls.

The problem of free harmonic vibrations of the fluid-filled elastic shell composed of a cylindrical part bounded by a hemispherical edge is considered. The shell is of uniform thickness $h$, and height $L$, made of homogeneous, isotropic material with elasticity modulus $E$, Poisson's ratio $v$ and mass density $\rho_{s}$. We designate a moistened shell surface by $S=S_{1}+S_{2}$, and a free surface by $S_{0}$. Surfaces $S_{1}$ and $S_{2}$ correspond to the spherical and cylindrical parts, respectively (Fig. 1).
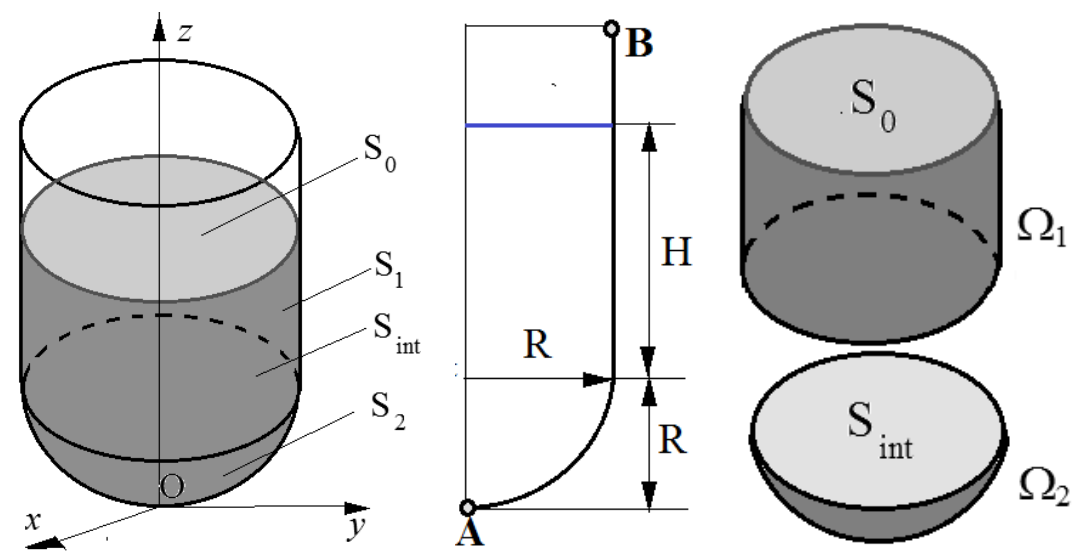

Figure 1: Shell structure, its sketch, and fluid sub-domains. 
Denote by $R$ radius of the spherical and cylindrical parts, and by $H$ a height of the wetted cylindrical part. Suppose the Cartesian coordinate system $0 x y z$ is connected with the shell, the liquid free surface $S_{0}$ coincides with the plane $z=H+R$ at the state of rest (Fig. 1). Let $\mathbf{U}=\left(U_{1}, U_{2}, U_{3}\right)$ denote the vector-function of shell displacements.

Assume that time dependent shell displacements are given by

$$
\mathbf{U}=\mathbf{u} \exp (i \Omega t) ; \quad \mathbf{u}=\left(u_{1}, u_{2}, u_{3}\right) .
$$

Here $\Omega$ is the vibration frequency; the time factor $\exp (i \Omega)$ will be omitted further on. After separation of the time factor, the vibrations of the shell without a liquid are described by a system of three partial differential equations

$$
\sum_{i=1}^{3} L_{i j} u_{i}=\Omega^{2} u_{j}, \quad j=1,2,3,
$$

where $L_{i j}$ are linear differential operators of Kirchhoff-Love shell theory (Levitin and Vassiliev [11]). The finite element method was applied by Ravnik et al. in [12] to evaluate the natural frequencies $\Omega_{k}$ and modes $\mathbf{u}_{k}, k=\overline{1, N}$ of the shell of revolution without a liquid. After forming the global stiffness $\mathbf{L}$ and mass $\mathbf{M}$ matrices, the following equation of motion for the shell containing fluid is obtained:

$$
\mathbf{L U}+\mathbf{M} \ddot{\mathbf{U}}=p_{d} \mathbf{n},
$$

where $\mathbf{n}$ is an external unit normal to the shell wetted surface, the term $p_{d} \mathbf{n}$ gives the fluid dynamical pressure upon the shell, normal to its surface.

To model the fluid domain, a mathematical model has been developed based on the following hypotheses: the liquid is incompressible and inviscid one, its motion is irrotational, only small vibrations (linear theory) need to be considered. So a scalar velocity potential $\Phi(x, y, z, t)$ whose gradient represents the fluid velocity can be introduced. The liquid pressure $p=p(x, y, z, t)$ acting on the wetted shell surface is obtained from the linearized Bernoulli's equation for a potential flow

$$
p=-\rho_{l}\left(\frac{\partial \Phi}{\partial t}+g z\right)+p_{0}, p_{s}=-\rho_{l} g z, p_{d}=-\rho_{l} \frac{\partial \Phi}{\partial t},
$$

where $g$ is the gravity acceleration, $z$ is the vertical coordinate of a point in the liquid, $\rho_{l}$ is the liquid density, $p_{s}$ and $p_{d}$ are static and dynamic components of the liquid pressure, $p_{0}$ is for atmospheric pressure.

At any instant the velocity potential $\Phi(x, y, z, t)$ could be determined from the following boundary value problem:

$$
\nabla^{2} \Phi=\frac{\partial^{2} \Phi}{\partial x^{2}}+\frac{\partial^{2} \Phi}{\partial y^{2}}+\frac{\partial^{2} \Phi}{\partial z^{2}}=0,\left.\frac{\partial \Phi}{\partial \mathbf{n}}\right|_{S}=\frac{\partial w}{\partial t},\left.\frac{\partial \Phi}{\partial \mathbf{n}}\right|_{S_{0}}=\frac{\partial \zeta}{\partial t} ; \frac{\partial \Phi}{\partial t}+\left.g \zeta\right|_{S_{0}}=0
$$

where $w$ denotes the normal component of the displacement vector $\mathbf{U}$, namely, $w=(\mathbf{U}, \mathbf{n})$; an unknown function $\zeta=\zeta(x, y, t)$ describes the form and position of the free surface. The second equation in (1) is the impermeability condition on the shell wetted surfaces, the 
third equation here is the kinematics boundary condition, which assumes that a fluid particle of the free surface will stay on this surface at any instance, the fourth equation in (1) is the dynamic boundary condition. It consists in equality of the liquid pressure on the free surface to atmospheric one. So the problem under consideration is reduced to the following system of differential equations:

$$
\mathbf{L U}+\mathbf{M} \ddot{\mathbf{U}}=p_{d} \mathbf{n} ; \quad \Delta \Phi=0
$$

with boundary conditions from (1), relative to $\Phi$, and fixation conditions of the shell, relative to $\mathbf{U}$. In this paper clamped-free (C-F) cylindrical-spherical shells are considered. i.e. shells of revolution are clamped at the ends A, and free at the ends B (Fig. 1).

Consider modes of fluid-filled shell vibrations in the form

$$
\mathbf{U}=\sum_{k=1}^{N} c_{k}(t) \mathbf{u}_{k},
$$

where $c_{k}(t)$ are unknown coefficients, and $\mathbf{u}_{k}$ are eigenmodes of the empty shell vibrations. Consider the potential $\Phi$ as $\Phi=\Phi_{1}+\Phi_{2}$, as it was done in [6]. The series for potential $\Phi_{1}$ can be written as

$$
\Phi_{1}=\sum_{k=1}^{N} \dot{c}_{k}(t) \phi_{1 k}
$$

Here time-dependant coefficients $c_{k}(t)$ are defined in eqn (3). To determine functions $\varphi_{1 k}$ the following boundary value problems is formulated:

$$
\Delta \phi_{1 k}=0,\left.\frac{\partial \phi_{1 k}}{\partial \mathbf{n}}\right|_{s}=w_{k},\left.\phi_{1 k}\right|_{S_{0}}=0, w_{k}=\left(\mathbf{u}_{k}, \mathbf{n}\right), k=\overline{1, N} .
$$

To determine potential $\Phi_{2}$ we have the problem of liquid vibrations in the rigid shell including gravity effects. Use the expansion

$$
\Phi_{2}=\sum_{k=1}^{M} \dot{d}_{k}(t) \phi_{2 k}
$$

where $d_{k} \cdot(t)$ are unknown coefficients, and functions $\varphi_{2 k}$ are natural modes of the liquid sloshing in the rigid tank. To obtain these modes the following boundary value problems are considered:

$$
\Delta \phi_{2 k}=0,\left.\frac{\partial \phi_{2 k}}{\partial \mathbf{n}}\right|_{S}=0 ; \frac{\partial \phi_{2 k}}{\partial t}+\left.g \zeta\right|_{S_{0}}=0 ;\left.\frac{\partial \phi_{2 k}}{\partial \mathbf{n}}\right|_{S_{0}}=\frac{\partial \varsigma}{\partial t}, k=\overline{1, N}
$$

The zero eigenvalue obviously exists for problem (5), but we exclude it with the help of the following orthogonality condition:

$$
\iint_{S_{0}} \frac{\partial \phi_{2 k}}{\partial \mathbf{n}} d S_{0}=0
$$

Finally, for the sum of potentials $\Phi=\Phi_{1}+\Phi_{2}$ the following expression is valid:

$$
\Phi=\sum_{k=1}^{N} \dot{c}_{k}(t) \phi_{1 k}+\sum_{k=1}^{M} \dot{d}_{k}(t) \phi_{2 k} .
$$

The unknown function $\zeta$ takes the form

$$
\zeta=\sum_{k=1}^{N} c_{k}(t) \frac{\partial \phi_{1 k}}{\partial \mathbf{n}}+\sum_{k=1}^{M} d_{k}(t) \frac{\partial \phi_{2 k}}{\partial \mathbf{n}} .
$$


Both boundary value problems (4) and (5) are reduced to systems of singular integral equations using a boundary element method in its direct formulation, Brebbia et al. [1]. Dropping indexes $1 k$ and $2 k$ the main relation for determining functions $\varphi_{1 k}$ and $\varphi_{2 k}$ can be written in the form

$$
2 \pi \phi\left(P_{0}\right)=\iint_{\sigma} q \frac{1}{\left|P-P_{0}\right|} d \sigma-\iint_{\sigma} \phi \frac{\partial}{\partial \mathbf{n}} \frac{1}{\left|P-P_{0}\right|} d \sigma,
$$

where $\sigma=S \cup S_{0}$, the value $r=\left|P-P_{0}\right|$ represents the Cartesian distance between a field point $P\left(x_{i}(P), \quad i=1,2,3\right)$ on the boundary $S$ and a collocation point $P_{0}\left(x_{i}\left(P_{0}\right), \quad i=1,2,3\right)$. The function $\varphi$, defined on the surface $S$, presents the pressure on the wetted shell surface, and the function $q$, defined on the surface $S_{0}$, is the flux, $q=\partial \phi / \partial \mathbf{n}$. There are two types of kernels in the integral operators introduced above

$$
\mathbf{A}\left(\sigma_{1}, \sigma_{2}\right) \psi=\iint_{\sigma_{1}} \psi \frac{\partial}{\partial \mathbf{n}} \frac{1}{\left|P-P_{0}\right|} d \sigma_{1} ; \quad \mathbf{B}\left(\sigma_{1}, \sigma_{2}\right) \psi=\iint_{\sigma_{1}} \psi \frac{1}{\left|P-P_{0}\right|} d \sigma_{1} ; \quad P \in \sigma_{1} ; P_{0} \in \sigma_{2},
$$

where $\frac{1}{\left|P-P_{0}\right|}$ and $\frac{\partial}{\partial \mathbf{n}} \frac{1}{\left|P-P_{0}\right|}$ are fundamental solution of the Laplace equation. It would be noted that if $\sigma_{1}=\sigma_{2}=\sigma$, then

$$
\mathbf{A}(\sigma, \sigma) \psi=2 \pi \mathbf{I} \psi+\iint_{\sigma} \psi \frac{\partial}{\partial \mathbf{n}} \frac{1}{\left|P-P_{0}\right|} d \sigma
$$

where $\mathbf{I}$ is the identity tensor.

So the problem of free harmonic vibrations of the fluid-filled elastic cylindricalspherical shell is reduced to defining the unknown potential and flux from relation (8) that can be rewritten as follows

$$
\mathbf{A}(\sigma, \sigma) \phi=\mathbf{B}(\sigma, \sigma) q
$$

\subsection{Axisymmetric elasticity problems for an isotropic body with an inhomogeneity}

Consider an elastic finite cylinder with an inhomogeneity. Suppose that the inhomogeneity has a form of a rounded cylinder (Fig. 2).

The external boundary of the body and its domain are denoted by $S$ and $\Omega_{1}$, respectively. The external boundary of the inclusion and its domain are denoted by $S_{\text {int }}$ and $\Omega_{2}$. The surface $S=\partial \Omega_{1}$ represents the boundary surface including inhomogeneity boundary $S_{\text {int }}$; so $S=S_{\text {int }} \cup S_{1}$, where $S_{1}$ is the cylinder boundaries, $S_{2}=S_{\text {int }}$ (Fig. 2).

Suppose that the body is statically loaded along the boundary $S$ by boundary tractions $\mathbf{t}$ and inside the domain by body forces $\mathbf{f}$.

This elastic cylinder with the inhomogeneity may be considered as a representative volume element for studying composites and nano-composites. Further, let $\Omega_{1}$ be the region of the matrix, and $\Omega_{2}$ be the region occupied by the inhomogeneity 

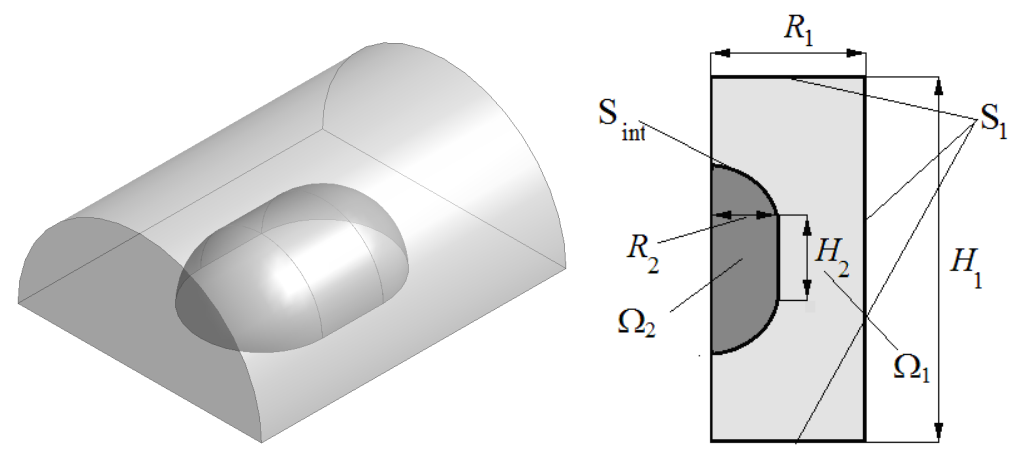

Figure 2: Elastic cylinder with an inclusion, its sketch, and sub-domains.

The three-dimensional isotropic elasticity equations state that the divergence of the stress tensor is zero

$$
\nabla \cdot \boldsymbol{\sigma}=\mathbf{f} ; \quad \boldsymbol{\sigma}=2 G[v /(1-2 v) \operatorname{tr}(\mathbf{e}) \mathbf{I}+\mathbf{e}] ; \quad 2 \mathbf{e}=\nabla \mathbf{u}+(\nabla \mathbf{u}) ; \quad \operatorname{tr}(\mathbf{e})=e_{i i},
$$

where $\mathbf{u}$ is the displacement vector, $\mathbf{e}$ is the infinitesimal strain tensor, $\sigma$ is the stress tensor, f is the body force, and $G=0.5 E /(1+v)$ is the shear modulus.

Let points $P_{0}, P$ and $Q$ are the collocation point, the points on the external boundary $S$ and inside the domain, respectively. The relation between the body tractions and its displacements can be expressed by the boundary integral equation (the Somigliana's identity) in the following form [1]:

$$
\mathbf{C u}=\iint_{\sigma} \mathbf{U}\left(P, P_{0}\right) \mathbf{t}(P) d \sigma-\iint_{\sigma} \mathbf{T}\left(P, P_{0}\right) \mathbf{u}(P) d \sigma+\iiint_{\Omega} \mathbf{U}(P, Q) \mathbf{f} d \Omega,
$$

where $\mathbf{C}$ is a constant matrix [8], that depends on the position of the point $\mathrm{P}$ on the surface $\sigma, \mathbf{U}\left(P, P_{0}\right)=\left\{U_{i j}\right\}$ and $\mathbf{T}\left(P, P_{0}\right)=\left\{T_{i j}\right\}$ are the Kelvin fundamental solutions of elastostatics

$$
\begin{gathered}
U_{i j}\left(P, P_{0}\right)=\frac{1}{16 \pi(1-v) G r}\left[(3-4 v) \delta_{i j}+r_{, i} r_{, j}\right], \\
T_{i j}\left(P, P_{0}\right)=\frac{1}{8 \pi(1-v) r^{2}}\left\{\left[(1-2 v) \delta_{i j}+3 r_{, i} r_{, j}\right] \frac{\partial r}{\partial \mathbf{n}}-(1-2 v)\left(r_{, i} n_{j}-r_{, j} n_{i}\right)\right\} .
\end{gathered}
$$

Here $r=\left(r_{i} r_{i}\right)^{1 / 2}, \quad r_{i}=x_{i}(P)-x_{i}\left(P_{0}\right), \quad r_{, i}=\partial r / \partial x_{i}(P)=r_{i} / r$.

Introducing the matrixes operators as in eqn (9)

$$
\mathbf{A}\left(\sigma_{1}, \sigma_{2}\right) \mathbf{u}=\iint_{\sigma_{1}} \mathbf{T}\left(P, P_{0}\right) \mathbf{u}(P) d \sigma_{1}, \quad \mathbf{B}\left(\sigma_{1}, \sigma_{2}\right) \mathbf{t}=\iint_{\sigma_{1}} \mathbf{U}\left(P, P_{0}\right) \mathbf{t}(P) d \sigma_{1}, P_{0} \in \sigma_{2},
$$

and taking into account that

$$
\mathbf{A}\left(\sigma_{1}, \sigma_{1}\right) \mathbf{u}=\mathbf{C u}+\iint_{\sigma_{1}} \mathbf{T}\left(P, P_{0}\right) \mathbf{u}(P) d \sigma_{1},
$$

we obtain the main relation in the absence of body forces, in the following form 


$$
\mathbf{A}(\sigma, \sigma) \mathbf{u}=\mathbf{B}(\sigma, \sigma) \mathbf{t} .
$$

The integral eqns (10), (11) for considered problems look the same. The important point here is that the singularity order at $r=0$ is precisely the same for both integral equations.

\section{MULTI-DOMAIN APPROACH}

Analytic solutions of the singular integral equations are restricted by simple geometrical shapes of the bodies, and by impossibility of studying cracks or inclusions, and thus it is necessary to use computational techniques. The proposed approach is relied on the boundary element method implementation in getting over the continuous models to their discrete analogues. For this purpose we use the multi-domain boundary element method, proposed by Brebbia et al. in [1].

Suppose that the integration domain $\Omega$ is divided into two parts $\Omega_{1}$ and $\Omega_{2}$ by the interface surface $S_{\text {int }}$ as in Figs 1,2 . Here $\partial \Omega_{i}=S_{\text {int }} \cup S_{i},(i=1,2)$.

Rewrite eqns (10), (11) in the form

$$
\mathbf{A}(\sigma, \sigma) \mathbf{X}=\mathbf{B}(\sigma, \sigma) \mathbf{Y}
$$

where vector $\mathbf{X}$ is for values of the potential (or displacements) in BEM mesh nodes, and $\mathbf{Y}$ is for values of the flux (or tractions). Introducing $\Sigma_{1}=S_{1}, \Sigma_{2}=S_{\text {int }}$, and $\Sigma_{3}=S_{2}$ allows us to obtain $A_{i j}=\mathbf{A}\left(\Sigma_{i}, \Sigma_{j}\right) ; \quad B_{i j}=\mathbf{B}\left(\Sigma_{i}, \Sigma_{j}\right), i, j=\overline{1,3}$. On the interface surface $S_{\text {int }}$ the following compatibility conditions are valid:

$$
\mathbf{X}_{S_{\text {int }} \cap \Omega_{1}}=\mathbf{X}_{S_{\text {int }} \cap \Omega_{2}}, \quad \mathbf{Y}_{S_{\text {int }} \cap \Omega_{1}}=-\mathbf{Y}_{S_{\text {int }} \cap \Omega_{2}} .
$$

Denote by $X_{i}$ the unknown values of $\mathbf{X}$ in nodes of $\Sigma_{i}$. The values of fluxes (tractions) are $Y_{i}$ $(i=1,2,3)$. By using the multi-domain approach [13] to determine unknown $\mathbf{X}$ values the next system of integral equations in the operator form is obtained:

$$
\begin{array}{ccc}
A_{11} X_{1}+A_{12} X_{2} & =B_{11} Y_{1}+B_{12} Y_{2}, & P_{0} \in \Sigma_{1}, \\
A_{21} X_{1}+A_{22} X_{2} & =B_{21} Y_{1}+B_{22} Y_{2}, & P_{0} \in S_{\text {int }} \cap \Omega_{1}, \\
A_{22} X_{2}+A_{23} X_{3}= & -B_{22} Y_{2}+B_{23} Y_{3}, & P_{0} \in S_{\text {int }} \cap \Omega_{2}, \\
A_{32} X_{2}+A_{33} X_{3}= & -B_{32} Y_{2}+B_{33} Y_{3}, & P_{0} \in \Sigma_{2} .
\end{array}
$$

Receiving $X_{2}$ from the second and third equations in (13) and substituting it into first and fourth equations respectively, one can obtain the next expressions:

$$
X_{1}=H_{11} Y_{1}+H_{12} Y_{2}, \quad X_{2}=H_{32} Y_{2}+H_{33} Y_{3},
$$

where $H_{i j}=(-1)^{i-j}\left(A_{i i}-A_{i 2} A_{22}^{-1} A_{2 i}\right)^{-1}\left(B_{i j}-A_{i 2} A_{22}^{-1} B_{2 i}\right), \quad i=1,3 ; j=1,2,3$. Subtracting third equation from the second one in (13) leads to the relation for $Y_{2}$

$$
\left(F_{1}+F_{3}\right) Y_{2}=G_{1} X_{1}+G_{3} X_{3}, G_{i}=(-1)^{i-2}\left(B_{2 i}-A_{2 i} H_{i i}\right), F_{i}=B_{22}+(-1)^{i-2} A_{2 i} H_{i 2}, i=1,3 .
$$

Note that system (14) only contains the unknowns $Y_{2}$ relative to the interface surface $S_{\text {int. }}$. The unknowns for other surfaces are expressed through $Y_{2}$ by given formulas. 


\section{BOUNDARY INTEGRAL EQUATIONS IN AXISYMMETRIC FORMULATION} Accordingly to the features of axisymmetric problems it is useful to transform the Cartesian coordinates system $(x, y, z)$ to a cylindrical one $(\rho, \theta, z)$ with following relations:

$$
x=\rho \cos \theta ; \quad y=\rho \sin \theta ; \quad z=z .
$$

The kernels of integral operators $\mathbf{A}$ and $\mathbf{B}$ for both potential and elasticity problems can be represented on terms of complete elliptic integrals [6], [10]. The basic procedure is to start with the standard boundary integral equations, replace Cartesian coordinates with cylindrical ones, and integrate with respect to $\theta$, taking into account that

$$
P=(\rho, \theta, z) ; \quad P=\left(\rho_{0}, \theta_{0}, z_{0}\right) ; \quad\left|P-P_{0}\right|=\sqrt{\rho^{2}+\rho_{0}^{2}+\left(z-z_{0}\right)^{2}-2 \rho \rho_{0} \cos \left(\theta-\theta_{0}\right)} .
$$

Then for integral operators of the potential theory problem we have

$$
\mathbf{A}(\sigma, \sigma)=2 \pi \mathbf{I}+A_{1}\left(P, P_{0}\right) \mathrm{E}(k)+A_{2}\left(P, P_{0}\right) \mathrm{K}(k) ; \quad \mathbf{B}(\sigma, \sigma)=B\left(P, P_{0}\right) \mathrm{K}(k) .
$$

Here

$$
\begin{gathered}
A_{1}\left(P, P_{0}\right)=\frac{4}{\sqrt{c} d}\left[\frac{\rho^{2}-\rho_{0}^{2}+\hat{z}^{2}}{2 \rho} n_{r}+\hat{z} n_{z}\right], A_{2}\left(P, P_{0}\right)=-\frac{2 n_{r}}{\rho \sqrt{c}}, B\left(P, P_{0}\right)=\frac{4}{\rho \sqrt{c}}, \\
\hat{z}=z_{0}-z, \quad a=\rho^{2}+\rho_{0}^{2}+\hat{z}^{2}, \quad b=2 \rho \rho_{0}, \quad c=a+b, \quad d=a-b, k^{2}=\frac{2 b}{a+b},
\end{gathered}
$$

$n_{r}, n_{z}$ are components of the external unit normal to the surface $\sigma$ in the $\rho$ and $z$ directions, respectively, and $\mathrm{E}(k), \mathrm{K}(k)$ are complete elliptic integrals

$$
\mathrm{E}(k)=\int_{0}^{\pi / 2}\left(1-k^{2} \sin ^{2} \psi\right)^{1 / 2} d \psi, \mathrm{K}(k)=\int_{0}^{\pi / 2}\left(1-k^{2} \sin ^{2} \psi\right)^{-1 / 2} d \psi
$$

The matrix integral operators of axisymmetric elasticity can be expressed in terms of complete elliptic integrals in the similar way

$$
\begin{gathered}
\mathbf{A}(\sigma, \sigma)=\mathbf{C}+\left\{T_{i j}\left(P, P_{0}\right)\right\}, \quad \mathbf{B}(\sigma, \sigma)=\left\{U_{i j}\left(P, P_{0}\right)\right\}, \quad i, j=\rho, z, \\
T_{i j}\left(P, P_{0}\right)=T_{i j}^{1} \mathrm{E}(k)+T_{i j}^{2} \mathrm{~K}(k), \quad U_{i j}\left(P, P_{0}\right)=U_{i j}^{1} \mathrm{E}(k)+U_{i j}^{2} \mathrm{~K}(k) .
\end{gathered}
$$

The displacement kernels here present weak singularities. These kernels are rearranged, so their components are following [10]:

$$
\begin{gathered}
U_{11}^{1}=\frac{-A}{\rho \rho_{0} \sqrt{c}}\left[(3-4 v) c+\hat{z}^{2} \frac{a}{d}\right], U_{11}^{2}=\frac{A}{\rho \rho_{0} \sqrt{c}}\left[(3-4 v) a+\hat{z}^{2}\right], U_{12}^{2}=\frac{A \hat{z}}{\rho_{0} \sqrt{c}}, \cdots U_{12}^{1}=-\frac{U_{12}^{2} h}{d}, \\
U_{21}^{2}=-\frac{A \hat{z}}{\rho \sqrt{c}}, \quad U_{21}^{1}=\frac{U_{21}^{2} f}{d}, \quad U_{22}^{2}=\frac{2 A(3-4 v)}{\sqrt{c}}, \quad U_{22}^{1}=\frac{2 A \hat{z}^{2}}{d \sqrt{c}}, \\
A=\left[16 \pi^{2} G(1-v)\right]^{-1}, \quad h=\rho^{2}-\rho_{0}^{2}+\hat{z}^{2}, \quad f=\rho_{0}^{2}-\rho^{2}+\hat{z}^{2} .
\end{gathered}
$$

Similarly, the traction kernels can be expressed as in [10] by following formulas 


$$
T_{i j}^{k}=2 G\left(T_{i j}^{k r} n_{r}+T_{i j}^{k z} n_{z}\right)
$$

where

$$
\begin{gathered}
T_{11}^{1 r}=\frac{A}{\rho_{0} \rho^{2} \sqrt{c} d}\left(2 v q+3 \rho^{2} f+\left(3 m-\frac{2 a^{2} f}{c d}\right) \hat{z}^{2}\right) ; T_{11}^{1 z}=\frac{-A}{\rho_{0} \rho \sqrt{c} d}\left[(2 v-3) a+\left(\frac{4 a^{2}}{c d}-3\right) \hat{z}^{2}\right] \\
T_{11}^{2 r}=\frac{A}{\rho_{0} \rho^{2} \sqrt{c} d}\left[2 v m-\frac{3}{2} n+\frac{a f \hat{z}^{2}}{c d}\right] ; T_{11}^{2 z}=\frac{A \hat{z}}{\rho \rho_{0} \sqrt{c}}\left(2 v-3+\frac{a \hat{z}^{2}}{c d}\right) ; \\
q=\frac{3 b^{2}}{2}+a r^{2}-2 a^{2} ; \quad m=2 a^{2}-\rho_{0}^{2} ; \quad n=2 \rho^{2}+3 \hat{z}^{2} ; \\
T_{12}^{1 r}=T_{11}^{1 z} ; T_{12}^{1 z}=\frac{A}{\rho_{0} \sqrt{c}}\left[(2 v-1) h+\left(\frac{8 f \rho_{0}^{2}}{c d}-1\right) \hat{z}^{2}\right] ; T_{12}^{2 r}=T_{11}^{2 z} ; T_{12}^{2 z}=\frac{A \hat{z}}{\rho \rho_{0} \sqrt{c}}\left(2 v-1+\frac{h \hat{z}^{2}}{c d}\right) ; \\
T_{21}^{1 r}=\frac{A}{d \sqrt{c}}\left[4(1+v)-\frac{a}{\rho^{2}}+\frac{8 a \hat{z}^{2}}{c d}\right], T_{21}^{1 z}=\frac{A}{d \sqrt{c}}\left[(1-2 v) f+\left(\frac{4 f a}{c d}-3\right) \hat{z}^{2}\right], T_{21}^{2 r}=\frac{A \hat{z}}{\sqrt{c}}\left(\frac{1}{\rho_{0}^{2}}+\frac{2 \hat{z}^{2}}{c d}\right) ; \\
T_{21}^{2 z}=\frac{A \hat{z}}{\rho_{0} \sqrt{c}}\left(2 v-1-\frac{f \hat{z}^{2}}{c d}\right) ; T_{22}^{1 r}=T_{21}^{1 z}, T_{22}^{1 z}=\frac{2 A \hat{z}}{d \sqrt{c}}\left(1-2 v+\frac{4 a \hat{z}^{2}}{c d}\right), T_{22}^{2 r}=T_{21}^{2 z}, T_{22}^{2 z}=\frac{2 A \hat{z}^{3}}{\rho_{0} \sqrt{c^{3}}} .
\end{gathered}
$$

The components $U_{i j}^{1,2}$ and $T_{i j}^{2}$ are regular functions. So the only singularity in these components is the logarithmic one due asymptotic behavior of $\mathrm{K}(k)$ for $k \rightarrow 1$. The special treatment is applied to evaluating the integrals using the orthogonal polynomials with logarithmic weights [1]. It was shown by Balas et al. [14] that components $T_{i j}^{1}$ contain terms $a_{1}=\left\lfloor n_{r}\left(\rho-\rho_{0}\right)+n_{z}\left(z-z_{0}\right)\right\rfloor / d, \quad \forall i, j$ and $a_{2}=\left[n_{z}\left(\rho-\rho_{0}\right)-n_{\rho}\left(z-z_{0}\right)\right] / d$ for $i \neq j$. As in the cylindrical coordinate system there are

$$
\rho=\rho(z), n_{z}=-\rho^{\prime} / \sqrt{1+\rho^{\prime 2}}, n_{r}=1 / \sqrt{1+\rho^{\prime 2}},
$$

then for $z \rightarrow z_{0}$ one can obtain that $a_{1} \rightarrow 0$, but $a_{2} \rightarrow\left[\left(z-z_{0}\right) \sqrt{1+\rho^{\prime 2}}\right]^{-1}$. Therefore, components $T_{11}^{1}, T_{22}^{1}$ are regular functions, whereas components $T_{12}^{1}, T_{21}^{1}$ have infinite gaps. For their numerical evaluation the method elaborated by Becker [15] is used.

\section{NUMERICAL EXAMPLES}

The proposed method is applied to solution of axisymmetric problems in potential theory and elastostatics.

\subsection{Validation of the proposed axisymmetric BEM}

The first step is to define the requisite number of boundary elements for evaluating unknown functions with given accuracy.

To testify the proposed numerical approach to potential theory problems the axisymmetric free liquid vibrations in a rigid cylindrical shell are considered. The circular cylindrical shell with a flat bottom has the following parameters: radius is $R=1 \mathrm{~m}$, the fluid filling level $H=2 \mathrm{~m}$. The numerical solution is obtained by using the BEM as it is 
described beforehand. In present numerical simulation we used 60 boundary elements along the bottom, 120 elements along wetted cylindrical parts and 60 elements along the free surface radius. Good agreement of analytical results for frequency parameter obtained by Ibrahim [16] and numerical ones received by proposed axisymmetric BEM is demonstrated in Table 1.

The example for validation in elastostatics is a long cylinder fixed at one edge $(z=0)$. The force $F$ is applied to the other edge $(z=L)$. The ratio of radius $R$ to the cylinder length $L$ is $1 / 10$, elasticity modulus is $E=2,11 \cdot 10^{6} \mathrm{MPa}$. The mesh used here has 120 boundary elements along the cylindrical part and 60 elements along top and bottom radii of the cylinder. This axisymmetric problem is solved using constant elements and integrations with 6 Gauss points. Table 2 shows the normalized displacements $w^{*}=w \pi E R^{2} / F L$ obtained at the points $r / R=0.05,0.25,0.5,0.75,0.99$ at $z=L$.

The presented BEM and analytical solution $w=F z / E \pi R^{2}$ are in very good agreement. The accuracy is decreased at boundary points $r / R=0.05$ and $r / R=0.99$. Calculations in these points require special treatment.

\subsection{Vibrations of compound cylindrical-spherical elastic shells}

Consider the fluid-filled elastic shell composed of a cylindrical part bounded by a hemispherical edge with thickness $h=0.01 \mathrm{~m}$, radius $R=1 \mathrm{~m}$, height $L=R+H=2 \mathrm{~m}$, elasticity modulus $E=2,11 \cdot 10^{6} \mathrm{MPa}$, Poisson's ratio $\nu=0.3$, mass density $\rho_{s}=8000 \mathrm{~kg} / \mathrm{m}^{3}$, and liquid density $\rho_{l}=1000 \mathrm{~kg} / \mathrm{m}^{3}$ (Fig. 1). At first stage the sloshing frequencies are evaluated for angular numbers $\alpha=0,1,2,4,5,6$. The results are presented in Table 3 .

Next stage is to evaluate the frequencies and modes of the elastic empty shell. The shell is supposed to be clamped over its rigid spherical bottom. In Table 3 there are frequencies of the empty cylindrical-spherical shell (CSS) described above and frequencies of an empty cylindrical shell (CS) clamped over its rigid flat bottom with radius $R=1 \mathrm{~m}$ and height $H=2 \mathrm{~m}$, evaluated for $\alpha=0,1,2,4,5,6$.

The numerical analysis demonstrates that the lowest frequencies are liquid sloshing ones, and occur for the angular number $\alpha=1$. Analyzing results of Table 3 one can observe, that the sloshing frequencies of cylindrical and cylindrical-spherical shells with equal heights are very close, whereas frequencies of these both empty and fluid-filled elastic shells with equal heights and radii of free surfaces are differed essentially.

Table 1: Comparison of analytical and numerical results.

\begin{tabular}{|l|c|c|c|c|c|}
\hline \multirow{2}{*}{ Method } & \multicolumn{5}{|c|}{ Frequency parameter $\omega^{2} / \mathrm{g}$} \\
\cline { 2 - 6 } & $n=1$ & $n=2$ & $n=3$ & $n=4$ & $n=5$ \\
\hline BEM & 1.833886 & 5.331447 & 8.536322 & 11.706103 & 14.864072 \\
\hline ANALYTICAL & 1.833885 & 5.331442 & 8.536316 & 11.706005 & 14.863589 \\
\hline
\end{tabular}

Table 2: Normalized static displacements $w^{*}$ of the elastic cylinder.

\begin{tabular}{|l|c|c|c|c|c|}
\hline \multirow{2}{*}{ Method } & \multicolumn{5}{|c|}{ Points, $r / R$} \\
\cline { 2 - 6 } & 0.05 & 0.25 & 0.5 & 0.75 & 0.99 \\
\hline BEM & 1.006 & 1.0003 & 1.00001 & 1.0004 & 1.02 \\
\hline Analytic & 1.0 & 1.0 & 1.0 & 1.0 & 1.0 \\
\hline
\end{tabular}


Table 3: Frequencies of empty and fluid-filled shells, Hz.

\begin{tabular}{|c|c|ccc|c|c|c|}
\hline$\alpha$ & $m$ & Sloshing CS & $\begin{array}{c}\text { Sloshing } \\
\text { CSS }\end{array}$ & Empty CS & $\begin{array}{c}\text { Empty } \\
\text { CSS }\end{array}$ & $\begin{array}{c}\text { Fluid- } \\
\text { filled CS }\end{array}$ & $\begin{array}{c}\text { Fluid- } \\
\text { filled CSS }\end{array}$ \\
\hline \multirow{4}{*}{0} & 1 & 6.1278 & 6.1283 & 398.132 & 796.263 & 145.582 & 221.553 \\
& 2 & 8.1217 & 8.2929 & 610.929 & 799.048 & 344.468 & 417.007 \\
& 3 & 9.9849 & 9.9871 & 810.703 & 817.043 & 398.132 & 512.361 \\
\hline \multirow{4}{*}{1} & 1 & 4.3494 & 4.2392 & 235.485 & 473.302 & 77.780 & 169.845 \\
& 2 & 7.2283 & 7.2291 & 606.710 & 779.754 & 348.210 & 389.965 \\
& 3 & 9.1463 & 9.1479 & 730.413 & 811.617 & 629.659 & 492.862 \\
\hline \multirow{4}{*}{2} & 1 & 5.4709 & 5.4708 & 117.679 & 290.119 & 49.489 & 136.445 \\
& 2 & 8.1076 & 8.1077 & 389.150 & 671.876 & 184.712 & 366.504 \\
& 3 & 9.8843 & 9.8861 & 619.164 & 774.231 & 319.253 & 483.943 \\
\hline \multirow{4}{*}{4} & 1 & 7.2188 & 7.2188 & 54.491 & 134.018 & 28.032 & 73.046 \\
& 2 & 9.5376 & 9.5383 & 186.299 & 426.793 & 100.414 & 251.088 \\
& 3 & 11.1482 & 11.1494 & 374.973 & 654.876 & 213.825 & 421.393 \\
\hline \multirow{3}{*}{5} & 1 & 7.9292 & 7.9286 & 65.136 & 110.755 & 35.401 & 60.490 \\
& 2 & 10.1535 & 10.1535 & 148.954 & 348.935 & 83.067 & 204.221 \\
& 3 & 11.7078 & 11.7082 & 300.608 & 592.120 & 176.017 & 379.170 \\
\hline \multirow{3}{*}{6} & 1 & 8.5739 & 8.5724 & 88.609 & 112.813 & 52.074 & 68.111 \\
& 2 & 10.7239 & 10.7227 & 139.468 & 300.132 & 83.090 & 188.162 \\
& 3 & 12.2322 & 12.3824 & 255.945 & 538.350 & 158.914 & 359.072 \\
\hline
\end{tabular}

The lowest frequency of the cylindrical shell caused by its elastic walls vibrations is nearly two times less than that one of the considered CSS. The frequencies of fluid-filled shell vibrations differ drastically from frequencies of empty ones for both shells. But with increasing the circumferential wave number $\alpha$ this difference become gradually smaller. Fig. 3 shows the modes of lowest frequencies.

For liquid sloshing the lowest frequencies corresponds to $\alpha=1$ (Fig. 3(a)), and for the elastic CSS vibrations it occurs at $\alpha=5$ (Fig. 3(b)).

\subsection{Stress-strain state of the elastic cylinder with the inhomogeneity}

Consider the cylinder fixed at one edge $(z=0.0)$, loaded uniformly at the other one $(z=5.0)$ and having the inclusion in the form of rounded cylinder (Fig. 2). Eqn (12) are perfect contact conditions. The ratio of cylinder and inclusion heights is $H_{1} / H_{2}=5$, and the ratio of their radii is $R_{1} / R_{2}=2$, elasticity moduli are $E_{1}=2,11 \cdot 10^{6} \mathrm{MPa}, E_{2}=2,11 \cdot 10^{7} \mathrm{MPa}$, and Poisson's ratios are $v_{1}=v_{2}=0.3$. We investigate the disturbance in a stress-strain state in the cylinder caused by the presence of this inhomogeneity. The multi-domain approach is applied here, and the resulting system of linear algebraic equations contains unknown displacements only along the interface surface $S_{\text {int }}$ (Fig. 2). Let now $\Sigma_{1}$ and $\Sigma_{2}$ are cylinder surface parts with given displacements and tractions, respectively, $\Sigma_{3}=\mathrm{S}_{\text {int }} \cap \Omega_{1}$, and $\Sigma_{4}=\mathrm{S}_{\mathrm{int}} \cap \Omega_{2}$. System (13) is rewritten for the case of given displacements at the elastic cylinder edge. Namely, we have

$$
-B_{i 1} Y_{1}+A_{i 2} X_{2}+A_{i 3} X_{3}-B_{i 3} Y_{3}=B_{12} Y_{2}, \quad P_{0} \in \Sigma_{i}, \quad A_{43} X_{3}+B_{43} Y_{3}=0, \quad P_{0} \in \Sigma_{4} .
$$




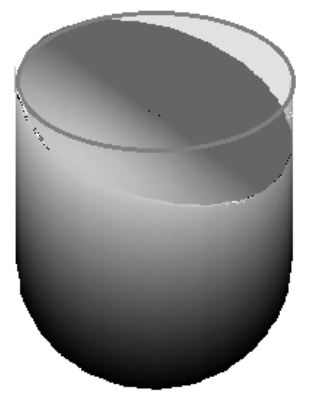

(a)

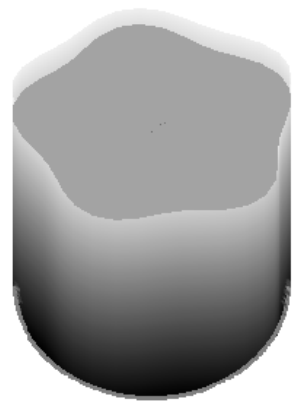

(b)

Figure 3: Modes of lowest frequencies.

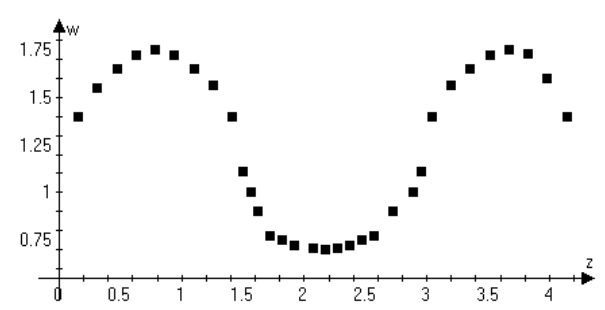

Figure 4: Displacements of interface surface.

So the relation between tractions and displacements of the cylinder on the interface surface is $Y_{3}=-B_{41}^{-1} A_{43} X_{3}$, and we obtain the final system of equations for the elastic cylinder with the inclusion. For numerical simulation we use following numbers $N_{i}$ of onedimensional boundary elements along surfaces $\Sigma_{I}(\mathrm{i}=1,2,3): N_{1}=150, N_{2}=30, N_{3}=240$. Fig. 4 shows the distributions of displacements in the elastic cylinder along the interface surface. In contrast to homogeneous cylinder the displacements are substantially nonuniform.

\section{CONCLUSION}

The effective method is elaborated for numerical solution of the boundary integral equations originated in axisymmetric problems of potential theory and elastostatics. Their form is received by using transition to cylindrical coordinates with following integration over angular variable. The approach is relied on the boundary element method implementation in getting from continuous models to their discrete analogues with thorough analysis of displacement and traction kernels. It reveals the similarities of the considered kernels in the singular integral operators that are represented on terms of complete elliptic integrals of first and second kinds both in potential and elasticity theories. Analysis of integrals proved the existence of logarithmic and Cauchy's singularities. The effective procedures for numerical treatment of these integrals taking into account the presence of these singularities are built. To verify the proposed method the numerical examples are provided. The coupled liquid and elastic walls vibrations of composed shells of revolution are considered to testify the proposed numerical approach to axisymmetric problems of potential theory. The long cylinder fixed at one edge and loaded by uniform stress at the other edge is considered for validation of the proposed method. The disturbance in a stress-strain state in the elastic cylinder caused by the presence of the inclusion is investigated with using the multi-domain approach.

\section{ACKNOWLEDGEMENTS}

Financial support for the project from STCU under grant No. 6247 is gratefully acknowledged. The authors would also like to thank our foreign collaborator Alexander Cheng, University of Mississippi, USA, for his constant support and interest to our research. 


\section{REFERENCES}

[1] Brebbia, C.A., Telles, J.C.F. \& Wrobel, L.C., Boundary Element Techniques: Theory and Applications in Engineering. Springer-Verlag: Berlin and New York, 1984.

[2] Wang, J. \& Gao, X.W., Structural multi-scale boundary element method based on element subdivision technique. Chinese Journal of Computational Mechanics, 27(2), pp. 258-263, 2010.

[3] Kane, J.H., Kashava Kumar, B.L. \& Saigal, S., An arbitrary condensing, noncondensing solution strategy for large scale, multi-zone boundary element analysis. Computer Methods in Applied Mechanics and Engineering, 79(2), pp. 21944, 1990. DOI: 10.1016/0045-7825(90)90133-7.

[4] Zhang, Ch., Cui, M., Wang, J., Gao, X.W., Sladek, J. \& Sladek, V., 3D crack analysis in functionally graded materials. Engineering Fracture Mechanics, 78, pp. 585-604, 2011.

[5] Gao, X.W. \& Yang, K., Thermal stress analysis of functionally graded material structures using boundary element method. Chinese Journal of Theoretical Chinese Journal of Theoretical and Applied Mechanics, 43(1), pp. 136-143, 2011.

[6] Gnitko, V., Degtyariov, K., Naumenko, V. \& Strelnikova, E., BEM and FEM analysis of the fluid-structure Interaction in tanks with baffles. International Journal of Computational Methods and Experimental Measurements, 5(3), pp. 317-328, 2017. DOI: $10.2495 / \mathrm{cmem}-\mathrm{v} 5-\mathrm{n} 3-317-328$.

[7] Rizzo, F.J. \& Shippy, D.J., A boundary integral approach to potential and elasticity problems for axisymmetric bodies with arbitrary boundary conditions. Mechanics Research Communications, 6(2), pp. 99-103, 1979.

DOI: 10.1016/0093-6413(79)90022-3.

[8] Cruse, T.A., Snow, D.W. \& Wilson, R.B., Numerical solutions in axisymmetric elasticity. Computers Structures, 7(3), pp. 445-451, 1977.

DOI: 10.1016/0045-7949(77)90081-5.

[9] Kermanidis, T.A., A numerical solution for axially symmetrical elasticity problems. International Journal of Solids and Structures, 11(4), pp. 493-500, 1975.

DOI: 10.1016/0020-7683(75)90084-0.

[10] Bakr, A.A., The Boundary Integral Equation Method in Axisymmetric Stress Analysis Problems, Springler Verlag: Berlin Heidelberg, New York, Tokyo, p. 213, 1986.

[11] Levitin, M. \& Vassiliev, D., Vibrations of shells contacting fluid: Asymptotic analysis. Acoustic Interactions with Submerged Elastic Structures, 5, pp. 310-332, 1996.

[12] Ravnik, J., Strelnikova, E., Gnitko, V., Degtyarev, K. \& Ogorodnyk, U., BEM and FEM analysis of fluid-structure interaction in a double tank. Engineering Analysis with Boundary Elements, 67, pp. 13-25, 2016.

[13] Gnitko, V., Degtyarev, K., Naumenko, V. \& Strelnikova, E., Coupled BEM and FEM Analysis of fluid-structure interaction in dual compartment tanks. International Journal of Computational Methods and Experimental Measurements, 6(6), pp. 976988, 2018.

[14] Balas, J., Sladek, J. \& Sladek, V., Stress Analysis by Boundary Elements. Studies in Applied Mechanics, Elsevier, 1989.

[15] Becker, A.A., The Boundary Element Method in Engineering-A Complete Course, McGraw-Hill, 1992.

[16] Ibrahim, R.A., Liquid Sloshing Dynamics: Theory and Applications, University Press: Cambridge, 2005. 